\title{
Gravitational Collapse of Colloidal Gels
}

\section{Citation}

Manley, S., J. M. Skotheim, L. Mahadevan, and D. A. Weitz. 2005. “Gravitational Collapse of

Colloidal Gels." Physical Review Letters 94 (21). https://doi.org/10.1103/physrevlett.94.218302.

\section{Permanent link}

http://nrs.harvard.edu/urn-3:HUL.InstRepos:41417294

\section{Terms of Use}

This article was downloaded from Harvard University's DASH repository, and is made available under the terms and conditions applicable to Other Posted Material, as set forth at http:// nrs.harvard.edu/urn-3:HUL.InstRepos:dash.current.terms-of-use\#LAA

\section{Share Your Story}

The Harvard community has made this article openly available.

Please share how this access benefits you. Submit a story.

Accessibility 


\title{
Gravitational Collapse of Colloidal Gels
}

\author{
S. Manley, J. M. Skotheim, L. Mahadevan, and D. A. Weitz \\ Department of Physics \& DEAS, Harvard University, Cambridge, Massachusetts 02138, USA
}

(Received 13 December 2004; published 3 June 2005)

\begin{abstract}
We present a unified framework for understanding the compaction of colloidal gels under their own weight. The dynamics of the collapse are determined by the value of the gravitational stress $\sigma_{g}$, as compared to the yield stress $\sigma_{Y}$ of the network. For $\sigma_{g}<\sigma_{Y}$, gels collapse poroelastically, and their rate of compression decays exponentially in time. For $\sigma_{g}>\sigma_{Y}$, the network eventually yields, leading to rapid settling. In both cases, the rate of collapse is backflow limited, while its overall magnitude is determined by a balance between gravitational stress and network elastic stress.
\end{abstract}

DOI: $10.1103 /$ PhysRevLett.94.218302

In the presence of attractive interactions, colloids can aggregate into ramified, fractal clusters, whose density decreases as their size increases. As a result, they can, in principle, grow to form a space-spanning network, or gel, at arbitrarily low volume fractions $\phi$. The networks formed through colloid aggregation are crucial for controlling the rheological properties of complex materials, including many food and personal care products; thus it is important to understand the limits of their stability. Ultimately, gel formation is constrained by gravity, which can impose a size-limiting strain on the aggregates [1], or drive sedimentation before a network can form [2]. Moreover, even after gels form, they can be mechanically unstable; at low $\phi$, or for weak interparticle attractions $U \sim k_{B} T$, they collapse to form a loose sediment at the bottom of the container. Network collapse is ubiquitous; it is observed for gels made from a variety of materials, and for a wide range of $U$ [2-9]. Qualitatively, collapse can occur smoothly, at a rate that decreases with time; alternatively, it can occur in distinct stages, characterized by a slow initial compression, after which gels undergo significant restructuring and rapid sedimentation, followed by a slow final compression to an equilibrium height. In the case of gels with a tunable depletion attraction with a short range, collapse dynamics switch from steady, or "creeping" sedimentation to three-stage, or "delayed" sedimentation as $U$ is decreased [6]. Collapse has been attributed to network aging properties for weak attractions $[3,6,8,9]$, or rheological properties for strong attractions [10]. However, no general framework has emerged that can account for the rich collapse phenomena that are common to so many systems.

In this Letter, we present a single framework that accounts for much of the diverse behavior observed in collapsing colloidal gels. We study the prototypical case of strongly attractive colloidal gels, whose structural and rheological properties are well characterized [11,12]. For gels formed in short sample cells and at high $\phi$, the height of the gel falls at a rate which decays exponentially with time, until it reaches a final equilibrium height, determined by the balance between the network elastic stress and the gravitational stress. For taller sample cells and lower $\phi$,
PACS numbers: 82.70.Dd, 61.43.Hv, 62.20.Fe, 82.70.Gg

gels initially exhibit the same behavior; however, they subsequently undergo a rapid, catastrophic collapse, which occurs because the network is ultimately unable to bear its own weight. At short times, the two cases are indistinguishable, and we show that the rate of collapse of the gel is limited by the backflow of the solvent through the solid network. Consistent with this, the initial rate of collapse decreases with increasing $\phi$, in a manner compatible with Darcy flow, independent of late-time behavior. We present a model for the collapse rate based on the theory of poroelasticity; this takes into account the resistance to compression arising from a combination of the fluid pressure and the elasticity of the network. For networks undergoing creeping sedimentation, the theory captures the full collapse behavior. For weakly elastic gels, the theory captures the initial collapse behavior; however, the details of the rapid collapse are more complicated, and are presumably linked to their nonlinear yield and shear-thinning properties.

We use aqueous solutions of charge-stabilized silica spheres, Ludox AS-40, with radius $a=10.5 \mathrm{~nm}$. To initiate aggregation, we add a divalent salt, $\mathrm{MgCl}_{2}$, to a final concentration of $20 \mathrm{mM}$; at this ionic strength particles experience a strong van der Waals attraction, and thus undergo primarily diffusion-limited cluster aggregation (DLCA) [13]. The resultant aggregates have a fractal dimension $d_{f} \sim 1.9$, with a size distribution sharply peaked about an average cluster size $R_{c}$. Clusters grow until $R_{c} \sim$ $a \phi^{1 /\left(d_{f}-3\right)}$, at which point they span space, and form a gel [11]. We study gels at a range of volume fractions, $10^{-3}<$ $\phi<10^{-2}$; the DLCA time for gelation at these concentrations is $<1 \mathrm{~min}$. The density difference between the particles and the solvent is $\Delta \rho=1.17 \mathrm{~g} / \mathrm{cm}^{3}$, which can result in gravitational stresses large enough to cause collapse in macroscopic samples. Experiments are performed in rectangular cells of height $h_{0}=3.5 \mathrm{~cm}$ and width $w=$ $2 \mathrm{~cm}$; cells are completely filled and sealed to ensure a horizontal interface, eliminating any effects of a curved meniscus. Cell depth was varied between $0.2-2 \mathrm{~cm}$, with no change in collapse behavior. The gels are imaged with a CCD camera to capture the collapse kinetics. 
As the samples settle, a sharp interface appears between the highly turbid gel and the clear supernatant. For all samples, there is an initial stage during which the collapse rate, $\nu_{0}$, is nearly constant; $\nu_{0}$ increases with decreasing $\phi$ as shown in Fig. 1 (inset). At the highest volume fractions, $\phi>3 \times 10^{-3}$, the collapse proceeds smoothly to the equilibrium height as shown in Fig. 1 where we plot the time evolution of the interface height $h(t)$. For $\phi<3 \times 10^{-3}$, there is also an initial slow collapse, but this is followed by a rapid collapse. This suggests that there is a common mechanism governing the initial collapse, independent of the long-time behavior.

In the initial stage of collapse, the network may be slowly compressing like a solid under its own weight; alternatively, it could be a fluid of aggregates which settle collectively [2]. To distinguish between these possibilities, we place a small plastic block of the same thickness but about half the width of the cell in the bottom corner of a sample chamber. As the top interface drops, a crack appears at the edge of the block, extending upwards through the gel; at the same time, the top interface is deformed, as shown schematically in Fig. 2 (inset). The appearance of this crack confirms that there is indeed an elastic network. The part of the network adjacent to the block is under stress; as it compresses, it creates a shear plane at the edge of the incompressible block, leading to crack formation. The difference in strains on the two sides persists through the gel, resulting in a step in the top interface which reflects the boundary condition at the bottom of the cell. Eventually, at the onset of the rapid collapse, the interface flattens indicating that the sample becomes a fluid as the gel is broken apart.

While the effects of gravitational stress are observable through the deformation of the solid network, the gels must expel fluid in order to compress. We hypothesize that the

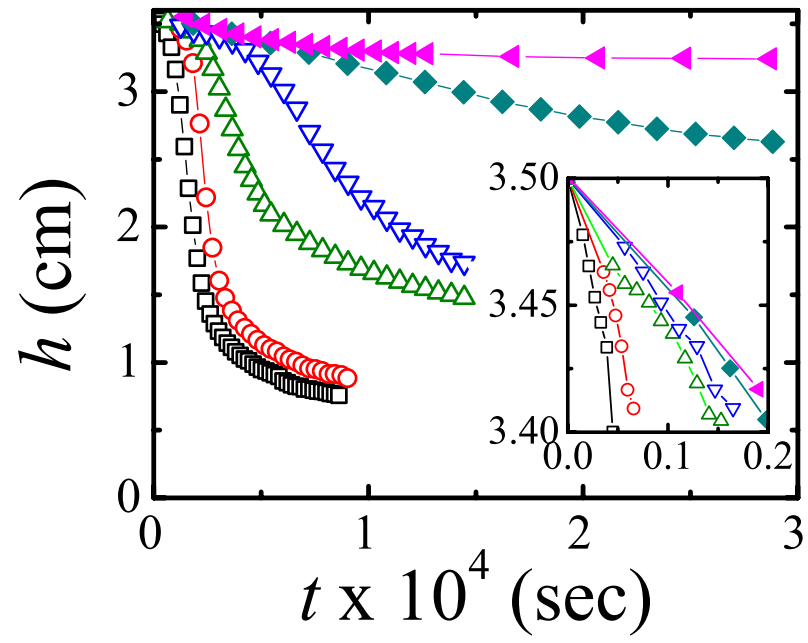

FIG. 1 (color online). Interface height as a function of time since initialization for $\phi=0.01(\square), 0.012(\bigcirc), 0.02(\triangle)$, $0.022(\nabla), 0.04(\diamond)$, and $0.08(\checkmark)$. Inset: blowup of the shorttime collapse. backflow of the fluid through the network limits the rate of compression of these gels, and hence determines their initial collapse. The interstitial fluid is under a pressure $P$ due to the weight of the gel; thus, it flows at a velocity $\nu$ within the porous and deformable network, leading to a local displacement of the solid of $w(z, t)$ in the $z$ direction. Using Darcy's law,

$$
(1-\phi)\left(\nu-\frac{\partial w}{\partial t}\right)=-\frac{k_{0}}{\eta} \frac{\partial P}{\partial z}
$$

where $k_{0}$ is the permeability of the gel and $\eta$ is the fluid viscosity. Because the rate at which fluid is expelled from a material element equals that rate at which solid enters,

$$
(1-\phi) \frac{\partial \nu}{\partial z}+\phi \frac{\partial^{2} w}{\partial z \partial t}=0 .
$$

Since $\phi \ll 1-\phi$, Eq. (2) implies that $\nu \approx 0$. At short times, the gel is uncompressed and the elastic stress is negligible, so that $\partial P / \partial z=-\Delta \rho g \phi$, and consequently Eq. (1) yields $k_{0}=-\frac{\eta}{\Delta \rho g \phi} \frac{\partial w}{\partial t}(t=0)$. To test this hypothesis, we use measurements of the initial velocity of the gel interface to determine the dimensionless permeability $k_{0} / a^{2}$ as a function of $\phi$, shown as open symbols in Fig. 2. We extend our range of $\phi$ by also performing experiments with larger silica spheres, Nissan MP-1040, with radius $a=50 \mathrm{~nm}$. We also plot previously published data from strongly aggregated calcium carbonate gels [2], and depletion-attracted colloid-polymer mixtures [6]. Remarkably, all of the data fall onto a single curve. To understand this, we compare the observed permeability with that derived from the Stokes's velocity for a

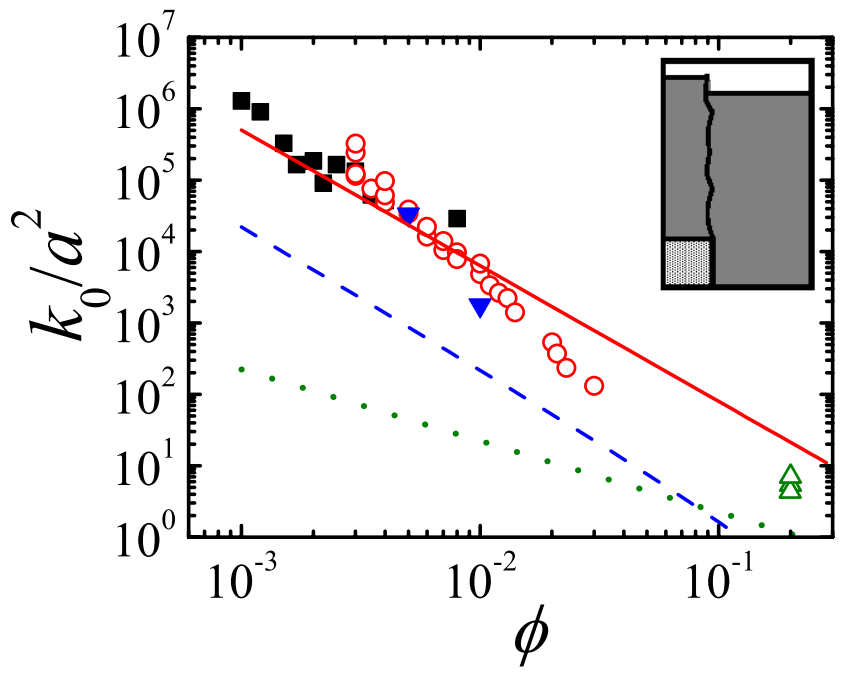

FIG. 2 (color online). Scaled permeability as a function of $\phi$, experiment values for: silica, $a=10 \mathrm{~nm}$ (ם); calcium carbonate, $a=35 \mathrm{~nm}(\bigcirc)$; PMMA, $a=186 \mathrm{~nm}(\triangle)$; silica, $a=$ $50 \mathrm{~nm}(\boldsymbol{\nabla})$. Permeability calculated using hard sphere model (dotted line), Carman-Kozeny model (dashed line), and cluster size (solid line). Inset: schematic of setup with light shaded block to test for presence of elastic network. 
suspension of isolated spheres, $k_{0}=\frac{2 a^{2}}{9 \phi}$ (dotted line), and that given by the semiempirical Carman-Kozeny relation, $k_{0}=\frac{(1-\phi)^{3} a^{2}}{45 \phi}$ [14], (dashed line). Here, the factor of 45 is an estimate of shape and tortuosity factors based on experimental results, and is reasonably successful for many types of granular materials, particularly at high $\phi$ [14]. Both expressions underestimate the experimental permeability. Instead, if we consider the characteristic pore size to be set by the largest length scale in the system, the cluster size $R_{c}$, the permeability is $k_{0} \sim \frac{a^{2}}{\phi^{2 /\left(3-d_{f}\right)}}$, with a prefactor of order 1 . This gives excellent agreement with all of the data, as shown by the solid line in Fig. 2. This scaling relationship is based on a more realistic description of the tenuous fractal geometry of the porous medium and thus, the good agreement with the data lends valuable physical insight as to the origin of the permeability. Furthermore, this confirms our hypothesis that the initial rate of collapse is determined by the backflow of the fluid.

At long times, contributions from the network elasticity must become important, since the deformation of the network is no longer negligible. At the highest volume fractions, $\phi>3 \times 10^{-3}$, the gel height smoothly approaches an equilibrium value, $h_{f}$, which is determined by the balance between gravitational stress and elastic forces. The gravitational load increases linearly with $\phi ; \sigma_{g} \sim \phi$, while the shear and bulk moduli increase as a power law, $G^{\prime} \sim \phi^{\nu}$, with exponent $\nu \sim 3.2-3.9[12,15,16]$. Thus, we expect that gravitational loading should result in smaller strains as $\phi$ is increased; this is confirmed by the data, as seen in Fig. 1.

At short times, the gravitational stress is balanced by the stresses due to fluid flow, while at long times it is balanced by the network elasticity. At intermediate times, we expect both to play a role. For Darcy flow the dominant contribution to the fluid stress comes from the fluid pressure, which is much larger than the viscous stresses. Thus, the constitutive equation for a poroelastic gel having an elastic modulus $E$ [17] is given by

$$
\sigma=E \frac{\partial w}{\partial z}-(1-\phi) P .
$$

The stress gradients in the gel are balanced by gravity so that

$$
\frac{\partial \sigma}{\partial z}=-\Delta \rho g \phi
$$

The initial displacement is zero, and at the lower boundary there is no displacement or fluid flux while at the upper boundary the hydrostatic pressure is negligible and the fluid is free to flow across the boundary. This provides the initial and boundary conditions,

$$
\begin{gathered}
w=0 \quad \text { at } t=0, \quad w=\frac{\partial P}{\partial z}=0 \quad \text { at } z=0, \\
E \frac{\partial w}{\partial z}=P=0 \quad \text { at } z=h(t) .
\end{gathered}
$$

The initial-boundary-value problem, Eqs. (1)-(5), is a variation of the problem of consolidation of a column of fluid-filled soil $[18,19]$ and may be solved using separation of variables, giving

$$
h_{0}-h(t)=\Delta h\left(1-e^{-t / \tau}\right),
$$

where the initial height is $h_{0}$, the total change in height is $\Delta h=\frac{\Delta \rho g \phi h_{0}^{2}}{2 E}$, and the time scale for the collapse is $\tau=\frac{4 \eta(1-\phi) h_{0}^{2}}{\pi^{2} k_{0} E}[20]$.

To test the validity of this prediction, we measure the collapse of samples in the creeping regime for a range of $h_{0}$. Experiments are performed in cylindrical cells; to access a wide range of heights, cells are only partially filled. The time evolution of $h(t)$ is well described by an exponential decay, consistent with Eq. (6), with a time constant that depends on initial height, as shown in Fig. 3 (top inset) for $\phi=5 \times 10^{-3}$. The characteristic time is well described by $\tau \sim h_{0}^{2}$, except for at the very tallest and the very shortest cells, where we see some deviations from the expected behavior. This scaling is in good agreement with the theoretical predictions. As a further consistency check, we calculate $E$ from the slope of the dashed lines shown in Fig. 3, and find $E \sim 4$ and $25 \mathrm{~Pa}$ for $\phi=5 \times$ $10^{-3}$ and $\phi=8 \times 10^{-3}$, respectively. As expected, the compressive moduli increase with $\phi$ as $E \sim \phi^{\nu}$, with $\nu \sim 4$; moreover, they are somewhat higher than the measured shear moduli at comparable $\phi$, as observed for other colloidal gels [16]. Since $\tau \sim \frac{\eta h_{0}^{2}}{k_{0} E}$ and $k_{0} \sim \phi^{-2}$, the curves in Fig. 3 collapse when $\tau$ is plotted against $h_{0}^{2} / \phi^{2}$ as shown in the bottom inset.

The deviation from the initial exponential collapse rate, observed for gels at the lowest $\phi$, must be the manifesta-

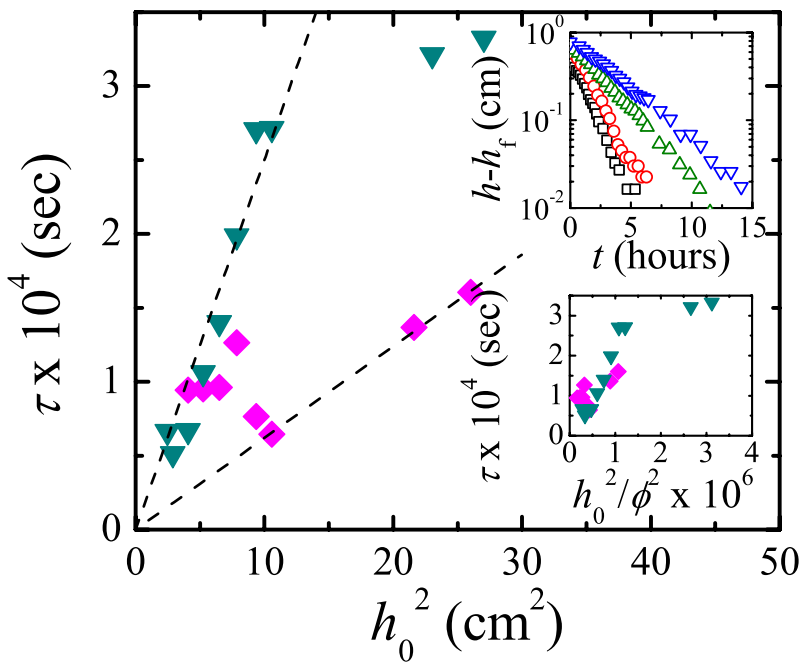

FIG. 3 (color online). Characteristic time scale for collapse as a function of initial height squared, for $\phi=5 \times 10^{-3}(\boldsymbol{\nabla})$ and $8 \times 10^{-3}(\checkmark)$. Top inset: $h(t)$ with baseline subtracted, for $\phi=$ $5 \times 10^{-3} ; h_{0}=1.8 \mathrm{~cm}(\square), 2.1 \mathrm{~cm}(\bigcirc), 2.4 \mathrm{~cm}(\triangle)$, and $2.7 \mathrm{~cm}$ $(\nabla)$. Bottom inset: characteristic time vs scaled height squared. 


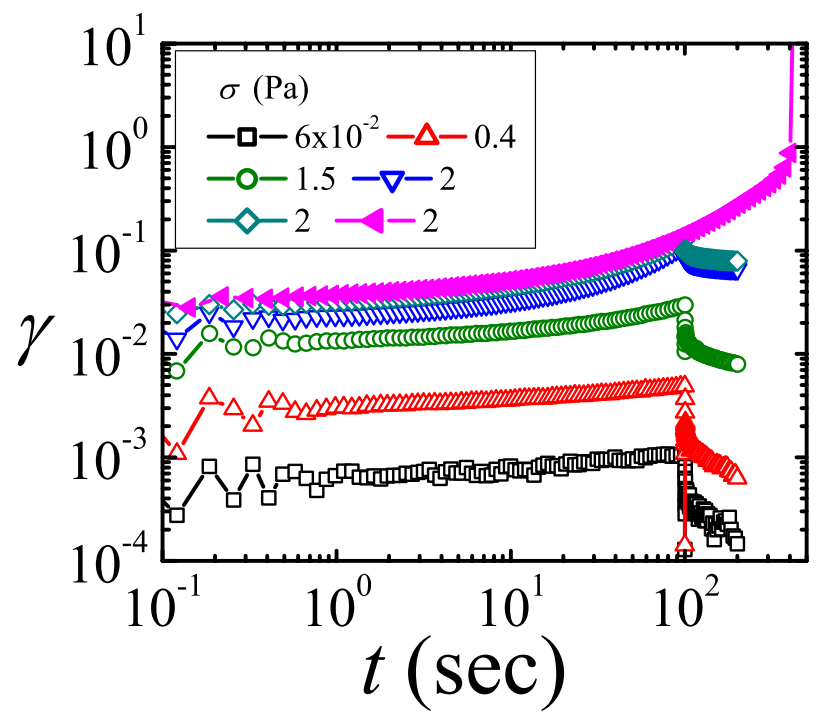

FIG. 4 (color online). Strain vs time after the application of increasingly large step stresses. The last $2 \mathrm{~Pa}$ step stress is applied for $600 \mathrm{sec}$, all previous stresses are applied for $100 \mathrm{sec}$.

tion of network failure, reflecting a crossover into the nonlinear viscoelastic regime. For the weakest gels, the gravitational stress is greater than the yield stress; however, the fluid bears most of the stress at short times, and the network initially deforms elastically. In this scenario, the time for network failure may be set by the time required for the gel to reach its yield strain. However, little is known about the nonlinear rheology of colloidal gels. To elucidate this behavior, we study the dynamics of network failure for a gel at $\phi=2 \times 10^{-2}$ in a stress-controlled rheometer, using a double-walled Couette geometry. We apply a step stress for 100 seconds, and then remove it, all while measuring the strain. For small stresses, $\sigma=6 \times 10^{-2} \mathrm{~Pa}$, the gel responds by deforming nearly instantaneously, with little additional deformation; it also recovers almost completely, as shown by the open squares in Fig. 4. By contrast, for $\sigma=1.5 \mathrm{~Pa}$, which is approaching the yield stress, the behavior is qualitatively different; the strain increases significantly, reflecting an increased strain rate as the gel begins to yield, as shown by the open circles in Fig. 4. However, the sample still recovers elastically when the stress is removed. The ultimate yielding of the gel is a slow process; we apply a stress of $2 \mathrm{~Pa} 2$ times; each time, the sample partially recovers. In fact, the gel only breaks and shear thins when the stress is applied for the third time, as shown by the solid symbols in Fig. 4. This experiment demonstrates that even when a network is overstressed, it can take a significant time to yield. This must be reflected in the collapse behavior of the low- $\phi$ gels, and may also play a role in the generic delayed collapse of gels [6].

The collapse of colloidal gels results from a balance between the gravitational load, their resistance to fluid flow, and their elastic and plastic properties. Given their elasticity, permeability, and geometry, their stability and collapse behavior under gravity can be predicted. Alternatively, by studying their collapse, it is possible to measure the elasticity of gels which are too weak to be measured with bulk rheological methods. Likewise, permeabilities that are too high to be measured with typical pressure filtration techniques can also be measured under gravity. The details of the collapse are captured by the theory of poroelasticity, which should be applicable for any elastic network immersed in a viscous background fluid, in different geometries, and under arbitrary stresses. The framework provided in this Letter correctly describes the behavior of the collapsing gels studied here; moreover, we expect that this framework will describe the delayed sedimentation of all forms of particle networks, independent of the specific details of their elasticity.

We would like to thank H. M. Wyss, A. J. C. Ladd, M. P. Brenner, V. Trappe, and E. R. Dufresne for helpful discussions. This work was supported by NASA (NAG3-2284), NSF (DMR-0243715), the Harvard MRSEC (DMR0213805), and the Norwegian Research Council.

[1] S. Manley et al., Phys. Rev. Lett. 93, 108302 (2004).

[2] C. Allain, M. Cloitre, and M. Wafra, Phys. Rev. Lett. 74, 1478 (1995).

[3] T. B. J. Blijdenstein et al., Langmuir 20, 11321 (2004).

[4] G. G. Glasrud et al., AIChE J. 39, 560 (1993).

[5] N.A.M. Verhaegh, D. Asnaghi, and H.N.W. Lekkerkerker, Physica (Amsterdam) 264A, 64 (1999).

[6] L. Starrs et al., J. Phys. Condens. Matter 14, 2485 (2002).

[7] J. R. Weeks, J. S. v. Duijneveldt, and B. Vincent, J. Phys. Condens. Matter 12, 9599 (2000).

[8] R. M. L. Evans and L. Starrs, J. Phys. Condens. Matter 14, 2507 (2002).

[9] M. L. Kilfoil et al., Phil. Trans. R. Soc. A 361, 753 (2003).

[10] D. Senis and C. Allain, Phys. Rev. E 55, 7797 (1997).

[11] M. Carpineti and M. Giglio, Phys. Rev. Lett. 68, 3327 (1992).

[12] T. Gisler, R. C. Ball, and D. A. Weitz, Phys. Rev. Lett. 82, 1064 (1999).

[13] D. A. Weitz et al., Phys. Rev. Lett. 53, 1657 (1984).

[14] J. Happel and H. Brenner, Low Reynolds Number Hydrodynamics (Kluwer, Dordrecht, 1991).

[15] A.H. Krall and D. A. Weitz, Phys. Rev. Lett. 80, 778 (1998).

[16] R. Buscall et al., J. Non-Newtonian Fluid Mech. 24, 183 (1987).

[17] For simplicity we have assumed that the Poisson ratio is zero.

[18] K. Terzaghi, Erdbaumechanik auf Bodenphysikalischer Grundlage (Deuticke, Leipzig, 1925).

[19] M. Biot, J. Appl. Phys. 12, 155 (1941).

[20] The complete solution is $h_{0}-h(t)=\Delta h(1-$ $\left.\sum_{n=0}^{\infty} \frac{4(-1)^{n}}{\pi^{3}(n+1 / 2)^{3}} e^{-(2 n+1)^{2} t / \tau}\right)$. We have dropped the higher, more rapidly decaying modes and fixed the first mode's prefactor so that $h(0)=h_{0}$. 